\title{
BECOMING A PHYSICIAN
}

\section{Tolerating Uncertainty — The Next Medical Revolution?}

Arabella L. Simpkin, B.M., B.Ch., M.M.Sc, and Richard M. Schwartzstein, M.D.

"A $t$ once it struck me what Aquality went to form a Man of Achievement . . . when a man is capable of being in uncertainties, mysteries, doubts, without any irritable reaching after fact and reason."

— John Keats, December $1817^{1}$

These words penned by John Keats, who was a physician as well as a poet, remind us of the human struggle to live in a grayscale space where uncertainty is rife - a space that is neither black nor white. Our quest for certainty is central to human psychology, however, and it both guides and misguides us.

Although physicians are rationally aware when uncertainty exists, the culture of medicine evinces a deep-rooted unwillingness to acknowledge and embrace it. Embodied in our teaching, our case-based learning curricula, and our research is the notion that we must unify a constellation of signs, symptoms, and test results into a solution. We demand a differential diagnosis after being presented with few facts and exhort our trainees to "put your money down" on a solution to the problem at hand despite the powerful effect of cognitive biases under these conditions. Too often, we focus on transforming a patient's gray-scale narrative into a black-and-white diagnosis that can be neatly categorized and labeled. The unintended consequence - an obsession with finding the right answer, at the risk of oversimplifying the richly iterative and evolutionary nature of clinical reasoning - is the very antithesis of humanistic, individualized patient-centered care.

We believe that a shift toward the acknowledgment and acceptance of uncertainty is essential - for us as physicians, for our patients, and for our health care system as a whole. Only if such a revolution occurs will we thrive in the coming medical era.

In medicine today, uncertainty is generally suppressed and ignored, consciously and subconsciously. Its suppression makes intuitive sense: being uncertain instills a sense of vulnerability in us - a sense of fear about what lies ahead. It is unsettling and makes us crave black-and-white zones, to escape this gray-scale space. Our protocols and checklists emphasize the black-andwhite aspects of medicine. Doctors often fear that by expressing uncertainty, they will project ignorance to patients and colleagues, so they internalize and mask it. We are still strongly influenced by a rationalist tradition that seeks to provide a world of apparent security.

Yet the reality is that doctors continually have to make decisions on the basis of imperfect data and limited knowledge, which leads to diagnostic uncertainty, coupled with the uncertainty that arises from unpredictable patient responses to treatment and from health care outcomes that are far from binary. Key elements for survival in the medical profession would seem, intuitively, to be a tolerance for uncertainty and a curiosity about the unknown. Have we created a culture that ignores and denies that requirement? Could our intolerance of uncertainty, in turn, be contributing to the accelerating rates of burnout and the rising cost of health care? For there is no doubt that absolute truth and certainty are hard to come by in clinical medicine.

Great tensions are created by the conflict between the quest for

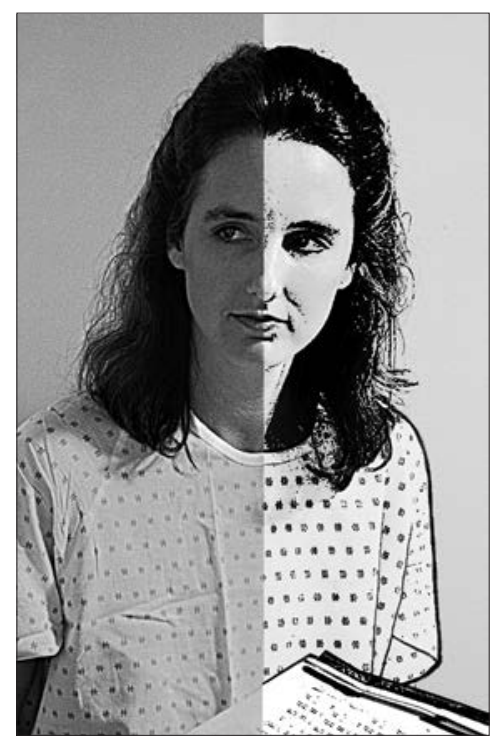

certainty and the reality of uncertainty. Doctors' maladaptive responses to uncertainty are known to contribute to work-related stress. ${ }^{2}$ Physicians' difficulty in accepting uncertainty has also been associated with detrimental effects on patients, including excessive ordering of tests that carry risks of false positive results or 
iatrogenic injury and withholding of information from patients. ${ }^{3}$ In addition, by attempting to achieve a sense of certainty too soon, we risk premature closure in our decision-making process, thereby allowing our hidden assumptions and unconscious biases to have more weight than they should, with increased potential for diagnostic error.

Our need to tolerate uncertainty has never been more urgent. Technology is advancing at lightning speed, and we are now able, need to thrive in 21st-century medicine.

We believe that cultivating a tolerance of uncertainty, and addressing the barriers to this goal for physicians, patients, and the health care system, will require a revolutionary change in medicine's cultural attitude and approach to uncertainty. Our curricula (formal, informal, and hidden), assessments, and evaluations will need to be modified to emphasize reasoning, the possibility of more than one right answer, and

\section{We can speak about "hypotheses" rather than "diagnoses," thereby changing the expectations of both patients and physicians and facilitating a shift in culture.}

at the touch of a button, to get instant access to a plethora of services and products. In our experience, many current medical students, the digital natives, seek structure, efficiency, and predictability; they insist on knowing "the right answer" and are frustrated when one cannot be supplied. This attitude no doubt increases the likelihood that they will perceive uncertainty as a threat. Given the growth of access to information online and electronically, students can spend less time at the bedside in the gray-scale world of medicine and more time in front of a screen absorbing processed and general information rather than immediate and idiosyncratic realities. Their online experience may reinforce their sense of a blackand-white world where certainty is readily achievable - the antithesis of the perspective they will consideration of our patients' values. Educators can start by asking questions that focus on "how" and "why," not "what" stimulating discussion that embraces the gray-scale aspects of human health and illness, aspects that cannot be neatly categorized, and encouraging students' curiosity to explore and capacity to sit comfortably with uncertainty, acknowledging that certainty is not always the end goal.

Our curricula should recognize diagnosis as dynamic and evolving - an iterative process that accounts for multiple, changing perspectives. We can speak about "hypotheses" rather than "diagnoses," thereby changing the expectations of both patients and physicians and facilitating a shift in culture. This shift may entail discussing uncertainty directly with patients, intentionally reflecting on its origins - subjec- tivity in the illness narrative, diagnostic sensitivity and specificity, unpredictability of treatment outcomes, and our own hidden assumptions and unconscious biases, to name a few. We can then teach physicians specifically how to communicate scientific uncertainty, which is essential if patients are to truly share in decision making, and we can reduce everyone's discomfort by reframing uncertainty as a surmountable challenge rather than as a threat.

In keeping with these curricular goals, our assessments of students can reflect the gray-scale environment, shifting away from the black-and-white multiplechoice questions that are all too common in our exams and that inculcate in students the belief that there is always a right answer. We need to focus on evaluating clinical reasoning and the demonstration of tolerance for uncertainty.

As we move further into the 21st century, it seems clear that technology will perform the routine tasks of medicine for which algorithms can be developed. Our value as physicians will lie in the gray-scale space, where we will have to support patients who are living with uncertainty - work that is essential to strong and meaningful doctor-patient relationships. It is therefore critical that we focus on thriving in this space and changing our professional culture to allow for uncertainty. As faculty, we will have to model for our students the practice of medicine in which it is all right to be uncertain - perhaps reminding ourselves of Osler's maxim that "medicine is a science of uncertainty and an art of probability." Ironically, only un- 
certainty is a sure thing. Certainty is an illusion.

Disclosure forms provided by the authors are available with the full text of this article at NEJM.org.

From the Division of General Internal Medicine, Massachusetts General Hospital (A.L.S.), Harvard Medical School (A.L.S.,
R.M.S.), and the Division of Pulmonary, Critical Care, and Sleep Medicine, Beth Israel Deaconess Medical Center (R.M.S.) all in Boston.

1. Forman MB. The letters of John Keats. Oxford, United Kingdom: Oxford University Press, 1931.

2. Logan RL, Scott PJ. Uncertainty in clinical practice: implications for quality and costs of health care. Lancet 1996;347:5958.

3. Kassirer JP. Our stubborn quest for diagnostic certainty: a cause of excessive testing. N Engl J Med 1989;320:1489-91.

4. Bean RB, Bean WB. Sir William Osler: aphorisms from his bedside teachings and writings. New York: Henry Schuman, 1950. DOI: 10.1056/NEJMp1606402

Copyright (c) 2016 Massachusetts Medical Society. 